\title{
Topology characterizing using packet forwarding distance dissimilarity in multi-greedy geographic routing
}

\author{
G. Oladeji-Atanda ${ }^{1, *}$ and D. Mpoeleng ${ }^{1}$ \\ ${ }^{1}$ Botswana International University of Science and Technology, Palapye, P/Bag 16, Botswana.
}

\begin{abstract}
Characterizing the topology of MANETs provides the means for packet routing protocols to perform adaptively and efficiently in the particular environments. We show that the geographic routing's greedy packet forwarding distance dissimilarity distributions in relation to node size characterizes MANET topologies and supports efficient multi-greedy forwarding. The models we described, based on the average greedy packet forwarding distance measures, showed distinct distribution patterns of the dissimilarity indices when applied to the example multi-greedy routing environment consisting of the ELLIPSOID and the GREEDY forwarding metrics. The scheme demonstrates the potential for adaptive forwarding performance to improve successful packets delivery in environments of high node-size variations such as VANETs.
\end{abstract}

Keywords: ad hoc networks, dissimilarity, distance learning, geographic packet forwarding, mobile environments, network topology, routing protocols.

Received on 02 October 2021, accepted on 06 January 2022, published on 11 January 2022

Copyright (C) 2022 G. Oladeji-Atanda et al., licensed to EAI. This is an open access article distributed under the terms of the Creative Commons Attribution license, which permits unlimited use, distribution, and reproduction in any medium so long as the original work is properly cited.

doi: 10.4108/eai.11-1-2022.172815

"Corresponding author: gbadebo.oladeji-atanda@studentmail.biust.ac.bw

\section{Introduction}

Characterizing the topology of a mobile ad hoc network (MANET) provides the means for packet routing protocols to perform adaptively and more efficiently in the environment [1] [2] [3]. The topology of a MANET is describable by the aggregates of its features including link durability, node degree, node velocity, node size or density, etc. Effective data communication by multihop packets relaying in a MANET is premised on a topology that has sufficiently and appropriately connected nodes, especially with the geographical greedy forwarding approach. In geographical routing, every intermediate node seeks to forward packets to a next relay neighbour that is closer in distance to the destination than itself. The geographical routing method has the advantage of minimal neighbour-table storage requirement at the nodes, unlike the traditional approach of maintaining network-wide routing tables. Hence, the geographical routing method is recommended for dynamic environments, such as the emergent flying ad hoc network (FANET) [4] and its counterpart vehicular ad hoc network (VANET) [5] [6], both of which exhibit high node mobility rates. FANETs and VANETs are also characterized by wide spatiotemporal variations in network node sizes. These dynamic network variations significantly impact routing protocol performance [7] [3] [6], which however can be improved through effective underlying topology characterizing and application of adaptive strategies [1].

Several fields of practice, including bio-informatics, transport systems, social networks, communication networks, etc., engage the dissimilarity approach to characterizing, differentiating, and classifying phenomena that exhibit collective similarity [2] [8] [9]. For example, in a social or a computer networks environment, communicating node pairs can be characterized based on the dissimilarity mapping of their message exchange rates [9]. The notion of dissimilarity is also referred to as distance in the sense of some quantifiable numerical difference between 
the objects of comparison [10] [11]. When not obvious in the objects of interest, the distance features for comparison can also be acquired from relevant data using some learning methods [10]. We propose the dissimilarity characterizing of dynamic MANET topologies using the packet forwarding distance [12], which is an integral feature in geographic routing operations. Packet forwarding distance is the weight attribute (typically the length measure) of the link between a node and its neighbour that is chosen as the next relay in packets routing toward a destination. The process of choosing the next relay node involves a greedy forwarding algorithm. The multi-greedy forwarding approach involves dynamically assigning the most suitable algorithm to the task of choosing the next relay node [13]. The greedy algorithms perform computations of geometric nature using relative locational and directional data of neighbour nodes to derive the applicable Euclidean distances for the choicemaking of a next relay at each forwarding step. Each of the geographical greedy routing algorithms, including ELLIPSOID, GREEDY, GREEDY-COMPASS, etc. have peculiar performance characteristics that further depends on the underlying network topology [7] [1] [14].

The main contribution of this paper is the proposal of MANET topology characterizing in geographic routing using the greedy packet forwarding distance dissimilarity measures in correlation to varying node size. Additionally, we show the applicability of the dissimilarity indices in multi-greedy forwarding for improving successful packets delivery through adaptiveness to the protocol performance indicators of the packets delivery rates (PDR), hop count, etc. PDR is the ratio of packets received at a destination compared to the amount sent from the source, which is a primary metric of routing procedures' effectiveness; while hop count is the number of relays that packets traverse, which influences delivery delays and network throughput rates. We demonstrate the potential of our proposal with the ELLIPSOID and the GREEDY methods in a VANET scenario as well as an adjoining MANET type.

In the next section, we discuss some related work on MANET topology characterizing and the dissimilarity approach. In section 3 we describe the geographic greedy routing method, with emphasis on the ELLIPSOID and the GREEDY forwarding metrics. In section 4 we explain the proposal of dissimilarity characterizing of topology and its potential for multi-greedy packet forwarding improvement. Section 5 shows simulation results of example topology characterizing using average packet forwarding distance dissimilarity distributions. Section 6 concludes the paper.

\section{Related work}

The characterizing of a network's topology is with the purpose of displaying some distinctive structural features of the comprised nodes and links in the environment. Network models of characterization based on the node and link measures of the degree distribution, clustering coefficient, dissimilarity indices, etc., have been applied to solving realworld problems including social collaborations, genetics, communication networks, etc. [2] [9]. For example, the authors in [4] investigated the proposal of airborne multihop packet-forwarding communication over dense airspace that also varies diurnally in node size. They [4] characterized the FANET topology based on the projected hop length and the hop count attributes of geographic routing to prescribe the transmission range parameters that would be efficient for that environment. In [5], the authors investigated the effectiveness of the Line-of-Sight (LoS) and the Non-Lineof-Sight (NLoS) packet inter-reception (PIR) time between communicating vehicular nodes in an urban scenario. They [5] characterized the VANET topology based on the distribution patterns of the LoS and NLoS PIR times, while the results led to their developing a 'context-aware beaconing' scheme to improve the NLoS PIR performance. Characterizing of networks is also aimed at topology control performance for efficiency in packet routing tasks such as in smart city management systems [15]. The authors in [15] proposed graph models that depict realistic urban topologies for intersections sensor node deployment design as well as for the evaluating of candidate routing methods. They [15] characterized the topologies of some selected cities, which led to the view that the gamma distribution pattern represents the deployment environment better than those of conventional random graphs, e.g. the Poisson distribution. Topology characterizing is quite often performed to learn an operating environment and to address adaptive routing [1] [14]. The authors in [1] did show that navigational goalfinding tasks over unknown (or changing) environments can be improved in efficiency through the search agent's learning of the underlying graph type and engaging the most suitable algorithmic strategy for future task performances. Three versions of the Depth First Search (DFS) algorithm were shown to have varied performance differences over the topologies of the Delaunay and the random graph [1]. The enhanced navigational search procedure can determine which DFS method to apply in a task by exploration sampling of the underlying arc lengths' histogram distribution patterns, which characterizes the different graph types by their dissimilarities. The topology characterizing scheme that we describe in this paper is similarly based on the statistical distributions of pertinent dissimilarity measures.

The dissimilarity approach to characterizing phenomena of interest relates to the fields of pattern recognition, machine learning and artificial intelligence. Dissimilarity characterizing of phenomenon involves the pairwise comparison of distinguishing structural feature(s) that the target objects possess based on some applicable distance measures, such as lexical distance, trust propagation distance, geographic distance, etc. [8] [9]. With the use of any suitable distance metric for a situation, the structural difference(s) of each of the comprised objects must be measured in pairwise comparisons to determine the relevant numerical dissimilarity values for statistical analysis. Dissimilarity characterizing to distinguish between collections of geometrical objects (points, lines, polygons, and polyhedrons) is discussed in [11]. Further dissimilarity metrics that the authors [11] itemized include the discrete 
distance, bottleneck distance, Minkowski distance, etc., for classifying, characterizing, and distinguishing between geometrical entities. The Minkowski distance with $\mathrm{p}=2$ is equivalent to the Euclidean distance, which is a primate measure in geographical routing computations. A review of approaches to learning of dissimilarity features based on matrix spaces and vectors is found in [10]. The dissimilarity characterizing of topology that we propose in this paper is based on the singular feature of greedy packet forwarding distance that is inherent in geographic routing, i.e. the length of a link involved with transmitting packets.

\section{Geographic greedy routing metrics}

The MANET topology is normally modelled as a graph $G(V, E)(t)$, where the set $V=\{v\}$ are the vertices or nodes and $E(t)=\left\{e_{u v}(t)=e_{v u}(t) \mid u, v \in V ; u \neq v\right\}$ are the existing edges or links at time $t$. Consider two linked neighbour nodes $u$ and $v$ with locational coordinates $\left(u_{x}, u_{y}\right)$ and $\left(v_{x}, v_{y}\right)$ in the 2-D plane; thus, the length of the link between them is the Euclidean distance defined by [16]:

$\operatorname{dist}(u, v)=\sqrt{\left(u_{x}-v_{x}\right)^{2}+\left(u_{y}-v_{y}\right)^{2}}$

We assume the unit disk network model of communication [16], such that for each node $u$ in the network, its neighbour set is defined as:

$N(u)=\left\{e_{u, v_{i}}\left|\operatorname{dist}\left(u, v_{i}\right) \leq r, \quad i=1,2, \ldots,\right| N(u) \mid\right\}$

where $r$ is a uniform nodal transmission radius. Nodes within distance $r$ of each other are neighbours and can communicate directly, otherwise, the exchange of data packets between them is by multihop forwarding. Each $v_{i} \in N(u)$ has a link with $u$. If $u$ directly sends packets to a neighbour $v_{i}$, the related distance coverage is a 'packet forwarding distance' [12]. We designate the occurrence of a greedy packet forwarding distance as ${ }^{2} d_{G F}$, defined to be the weight in terms of the length measure of the concerned link or edge such that we have

$$
d_{G F}\left(e_{u, v_{i}}(t)\right)=\operatorname{dist}\left(u, v_{i}\right)(t) .
$$

Figure 1 depicts a geographic greedy packet forwarding step that requires the node $u$ to choose a next relay neighbour from the subset $N(u)^{\prime}$ in the progress region, i.e. $N(u)^{\prime} \subseteq$ $N(u)$ consisting of $v_{1}, v_{2}$, and $v_{3}$. Greedy forwarding fails if $N(u)^{\prime}$ is empty, and we say a local maximum occurred [4] [6]. Different methods, such as embedded graph planarization routing [6] and the ${ }^{3}$ Ellipsoid approach [17] have been designed to handle recovery from greedy failure.

\footnotetext{
${ }^{2}$ In [4], the hop distance of greedy forwarding is the number of relays a packet traverses and is designated $h_{G F}$. It is also called link distance [14] and hop count [12].
}

${ }^{3}$ ELLIPSOID derived its name from its 3-D version mode of operation [17]
But in this paper, we are addressing efficiency improvement of progress forwarding only. Thus, excluding local maxima, the greedy forwarding step (depicted in Figure 1) is repeated at each intermediate node until the destination is reached.

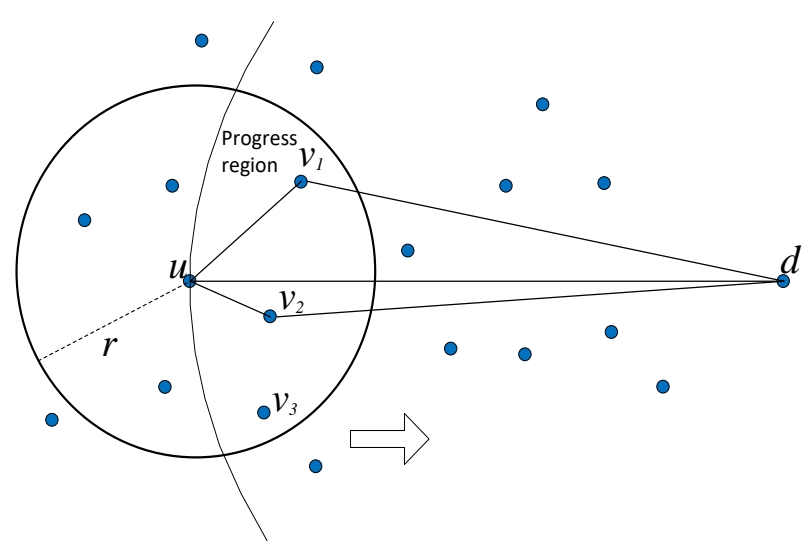

Figure 1. A geographic greedy forwarding step by which the node $u$ must choose a next relay neighbour from the set $\left\{v_{1}, v_{2}\right.$ or $\left.v_{3}\right\}$ toward the destination $d$.

The algorithms that implement greedy forwarding perform the next packet relay neighbour selections differently and are also influenced by the network topology [1] [4] [7]. In this paper, we show that the GREEDY and the ELLIPSOID [16] [14] methods' packet forwarding distance measures can be aggregated for dissimilarity characterizing of topology to aid adaptive multi-greedy [13] geographic routing performance in varying node size environments. From Figure 1 and based on (1), the GREEDY metric is defined as [16]:

$$
\begin{aligned}
& \min \{\operatorname{dist}(v, d)\} \\
& =\min \left\{\sqrt{\left(v_{x}-d_{x}\right)^{2}+\left(v_{y}-d_{y}\right)^{2}}\right\}
\end{aligned}
$$

The GREEDY algorithm evaluates nodes in the progress region for a relay neighbour having the least Euclidean distance to the destination. On the other hand, the ELLIPSOID method evaluates for a relay having the least value in the Euclidean distances of the link to the neighbour and on to the destination. The ELLIPSOID metric is defined as [16] [17]:

$$
\begin{gathered}
\min \{\operatorname{dist}(u, v)+\operatorname{dist}(v, d)\} \\
=\min \left\{\sqrt{\left(\left(u_{x}-v_{x}\right)^{2}+\left(u_{y}-v_{y}\right)^{2}\right)}\right. \\
\left.+\sqrt{\left(\left(v_{x}-d_{x}\right)^{2}+\left(v_{y}-d_{y}\right)^{2}\right)}\right\}
\end{gathered}
$$


Based on the scenario of Figure 1, the next relay neighbour choice of GREEDY will be $v_{1}$ having the distance $\operatorname{dist}\left(v_{1}, d\right)$ that is apparently the least to the destination in comparison to those of $v_{2}$ or $v_{3}$. But the ELLIPSOID choice would be $v_{2}$ because the distance $\operatorname{dist}\left(u, v_{2}\right)+\operatorname{dist}\left(v_{2}, d\right)$ is apparently the least compared to those of $v_{1}$ or $v_{3}$. Note that the ELLIPSOID neighbour choice $v_{2}$ is closer to the forwarding node $u$ in comparison to $v_{1}$ of the GREEDY metric; generally implying that $\operatorname{dist}(u, v)^{(\text {ELLIPSOID })} \leq$ $\operatorname{dist}(u, v)^{(G R E E D Y)}$ is an invariant when we compare these in a greedy forwarding step. This also implies that the end-toend packet delivery hop count [12] [4] values of ELLIPSOID would be higher or equal to the corresponding ones for GREEDY in routing ratings. The GREEDY method is popularly used in geographic routing for its efficient packet delivery performances, but the ELLIPSOID method is also noted in the same perspective [16] [14] [17]. Invariably, with the ELLIPSOID and the GREEDY forwarding methods, we want to answer the following questions.

(i) What relationship exists between the greedy packet forwarding distance and network node size?

(ii) How does the packet delivery performance of the greedy methods in geographic routing compare to the relationship mentioned in (i) above?

\section{Dissimilarity characterizing of topology in multi-greedy packet forwarding}

The 'greedy packet forwarding distance' is the dissimilarity feature that we adopt for the MANET topology characterizing proposal, with multi-greedy routing. Multiple optimally performing metrics are adaptively applied to packet forwarding in multi-greedy routing [13].

\subsection{The dissimilarity metric}

For any two comparable entities $a_{i}$ and $a_{j}$ possessing some structural difference, the dissimilarity measure takes on a value from 0 (no dissimilarity) to any positive numerical magnitude, i.e. $[0, \infty]$. The higher the value, the more the significance of dissimilarity distinction between the target pairwise objects. This is unlike the 'similarity' measure that varies between the range [0,1], where also 0 means no similarity but values tending to 1 implies significant similarity.

The Euclidean distance of dissimilarity, which compares $n$-dimension of attributes on objects $a_{i}$ and $a_{j}$, is defined as [2] [10]:

$$
D\left(a_{i}, a_{j}\right)=\sqrt{\sum_{k=1}^{n}\left(a_{i k}-a_{j k}\right)^{2}} .
$$

Since, we shall be dealing with a single attribute, i.e. average length of the packet forwarding distance, (6) reduces to finding the difference between any two given lengths:

$$
\begin{gathered}
D\left(a_{i}, a_{j}\right)=\sqrt{\left(a_{i}-a_{j}\right)^{2}} \\
=\left|a_{i}-a_{j}\right|
\end{gathered}
$$

Note the distinction between 'feature distance or dissimilarity' that distinguishes the comparative structural difference between any pairwise objects of interest, and the 'geographical distance' that describes the length between any two locations. Nevertheless, we employ the Euclidean distance metric for both measures. Our dissimilarity metric computes the difference between any two given length measures of the packet forwarding distance.

\subsection{Dissimilarity based on averages of the greedy packet forwarding distance}

In this paper, we refer only to the length attribute of a link in designating the packet forwarding distance as specified by (3). Nevertheless, the link attribute of length may be corroborated by others, such as link durability, packets reception rates, PIR [5], etc. The packet forwarding distance generated in geographic routing generally satisfy the properties specified for a dissimilarity metric [11], which are (i) $\operatorname{dist}(a, a)(t)=0$, (ii) $\operatorname{dist}(a, b)(t)=0$ implies $a=b$, and (iii) $\operatorname{dist}(a, b)(t)+\operatorname{dist}(a, c)(t) \geq \operatorname{dist}(b, c)(t)$, at any time instance $t$.

Using (3), the set

$$
D_{G F}=\left\{d_{G F_{i}}(t)|i=1,2,3, \ldots,| D_{G F} \mid\right\}
$$

contains unique packet forwarding distance elements, having different time stamps $t$, even when some are of equal length values. For convenience, we shall suspend including $t$ on the terms we express.

Hence, we can define the dissimilarity metric for comparing the packet forwarding distance averages generated in geographic routing performances. Additionally, with respect to the peak node-size measure of a network, we specify the differential factor that depicts the ratios of the dissimilarity values.

(i) Dissimilarity of the average packet forwarding distance

Using (3) and (8), the averaging of the values of the greedy packet forwarding distance is performed by:

$$
\bar{d}_{G F}=\frac{1}{\left|D_{G F}\right|} \sum_{i=1}^{\left|D_{G F}\right|} d_{G F_{i}}
$$

Let $\bar{d}_{G F}^{(\text {ELLIPSOID })}$ represent the average of the packet forwarding distance sampling for the ELLIPSOID method 
usage in a network, and the corresponding $\bar{d}_{G F}^{(G R E E D Y)}$ represent that of the GREEDY method. In this paper, to avoid mix up of the notation ' $\mathrm{D}$ ' in (7) and (8), we specify the dissimilarity metric function $\operatorname{ddist}($.) for the ELLIPSOID and the GREEDY averages, using (7) and (9) as:

$$
\begin{aligned}
& \operatorname{ddist}\left(\bar{d}_{G F}^{(E L L I P S O I D)(k)}, \bar{d}_{G F}^{(G R E E D Y)(k)}\right) \\
= & \left|\bar{d}_{G F}^{(E L L I P S O I D)(k)}-\bar{d}_{G F}^{(G R E E D Y)(k)}\right|
\end{aligned}
$$

which also distinguishes the comparable pairwise averages by their node size $k$.

(ii) Differential Factor

We specify the differential factor function $d f($.$) . Let b^{(k)}$ be the dissimilarity value obtainable for a $k^{\text {th }}$ node size of the network, and $b^{(m)}$ that of the peak size, $0 \leq k \leq m$, then :

$$
d f\left(b^{(k)}, b^{(m)}\right)=\frac{b^{(m)}}{b^{(k)}}
$$

which, based on (10), becomes:

$$
\begin{gathered}
d f\left(\begin{array}{l}
\operatorname{ddist}\left(\bar{d}_{G F}^{(E L L I P S O I D)(k)}, \bar{d}_{G F}^{(G R E E D Y)(k)}\right), \\
\operatorname{ddist}\left(\bar{d}_{G F}^{(E L L I P S O I D)(m)}, \bar{d}_{G F}^{(G R E E D Y)(m)}\right)
\end{array}\right) \\
=\frac{\left|\bar{d}_{G F}^{(E L L I P S O I D)(m)}-\bar{d}_{G F}^{(G R E E D Y)(m)}\right|}{\left|\bar{d}_{G F}^{(E L L I P S O I D)(k)}-\bar{d}_{G F}^{(G R E E D Y)(k)}\right|}
\end{gathered}
$$

The differential factor allows us to see at a glance the comparative magnitude of the dissimilarity values, and consequently, determine if it is advantageous or not to employ a performance improvement scheme such as the multi-greedy [13] forwarding. High margins of differences in the differential factors reflect a MANET environment where multi-greedy forwarding could be advantageous. We have involved two greedy methods, namely ELLIPSOID and GREEDY, in our topology characterizing models. Nevertheless, these models can be altered to include some different or increased number of greedy metrics depending on the situation.

\subsection{Multi-greedy forwarding application of the dissimilarity indices}

The multi-greedy approach to improving packet forwarding efficiency is described in [13]. The approach is necessitated by the realization that the greedy metrics outperform each other over different topological conditions [7] [1]. It involves the adaptive engagement of optimally performing greedy algorithms in an environment to improve packet routing tasks. In multi-greedy routing, multiple greedy algorithms $\left\{x_{1}, \ldots, x_{n}\right\}$ are engaged and selectively applied to packet forwarding one at a time depending on the optimal $x_{j}$ one that matches each situation, determined by the function [13]:

$$
\underset{x_{j} \in x}{\text { optimize }} y=f(x)
$$

For a packet forwarding step, the algorithm, i.e. an $x_{j}$, that selects a next relay node, is itself selected thus [13]:

$$
f\left(x_{1}, \ldots, x_{n}\right)=\mathrm{V}_{1}^{n} x_{j}
$$

For a dissimilarity value $b^{(k)}$ associated with a $k^{\text {th }}$ node-size and with respect to protocol PDR and hop count performance indications in multi-greedy method assignments, (14) yields:

$$
\begin{aligned}
& f\left(x_{1}, \ldots, x_{n}\right)=\max _{P D R\left(b^{(k)}\right)}\left\{x_{j}\right\}, \\
& f\left(x_{1}, \ldots, x_{n}\right)=\min _{\text {hopcount }\left(b^{(k)}\right)}\left\{x_{j}\right\}
\end{aligned}
$$

Similar to (15) and (16), we may specify for the protocol performance indicators of delay, etc., as well. Any greedy method thus assigned performs the next relay neighbour selection and should commensurately contribute to optimally efficient packets delivery performance.

A geographic routing protocol, such as the GPSR [6], can be enhanced to periodically sample packet forwarding distance dissimilarity values for the characterizing of a network's prevailing topology. Accordingly, the protocol could assign the engaged greedy metrics to packet forwarding performance by their optimality ratings in relation to the current topology dissimilarity value or node size.

\section{Simulation performance and results}

We demonstrate the packet forwarding distance dissimilarity characterizing of topology with simulated VANET and MANET scenarios. The VANET topology is an example of MANETs that characteristically vary widely in node size [4] [15]. We introduced the MANET environment, with a wider band of node sizes, as an adjoining example to validate the dissimilarity characteristics of the greedy packet forwarding distance measures. We employ the GPSR [6] routing protocol with which we engage the ELLIPSOID and the GREEDY packet forwarding metrics. The VANET data, of Krauss' mobility model, together with the GPSR code that we used are made available through [6]. The VANET environment's range of 30-110 node sizes enabled us to concisely demonstrate the packet forwarding distance dissimilarity phenomenon. The mobility model of the MANET environment is the Random Way Point (RWP) implemented with a wide 20-560 range of node sizes. The results show the effectiveness of the dissimilarity approach 
to topology characterizing and its applicability to efficient packet forwarding in the multi-greedy scheme.

\subsection{Simulation setup}

The simulation parameters that we used in the topology characterizing experiments are shown in Table 1. We used the NS-3 simulator for running the GPSR routing protocol in the VANET and the MANET environments. The NS-3 is incapable of handling dynamically varying node sizes; therefore, we ran simulations for the different node sizes separately but with all the other parameters fixed. We disabled the greedy failure recovery mode of the GPSR since our focus is on the progress forwarding performance of geographic routing. We used the BonnMotion [18] tool to generate the RWP mobility model for the MANET input to the NS-3 simulator. The node speeds for both the VANET and the MANET mobility was set at $0-15 \mathrm{~m} / \mathrm{s}$. The simulation time that we set was for $200 \mathrm{~s}$. We note that different simulation running times produce different PDR performance results patterns for both ELLIPSOID and GREEDY since the measure is based on periodical rates. For instance, our experimenting with the 600s simulation period results in a monotonically increasing pattern of the PDR outcome for both greedy methods. Nevertheless, the works in [16] [19] show the PDR behaviour of nonmonotonically increasing sort; especially in [7] where different topologies display varied outcomes. In general, the network scenarios where PDR, hop count and delay exhibit unevenness in the outcomes of protocol performance could be leveraged for improvement by the multi-greedy application. It is shown in [7] that the PDR and the hop count can vary by as much as $25 \%$ due to changes in network configurations. Therefore, in a network where the protocol performance patterns appear exploitable, then the multi-greedy method can be engaged for packet delivery improvement.

Table 1. Simulation parameters

\begin{tabular}{ll}
\hline \multicolumn{1}{c}{ Parameter } & \multicolumn{1}{c}{ Value } \\
NS-3 simulator & v. 3.23 \\
Routing protocol & GPSR \\
No of nodes & $30-110$ (Krauss), 20-560 (RWP) \\
Simulation area & $1100 \mathrm{~m}^{2}$ (VANET), 2000m ${ }^{2}$ (MANET) \\
Simulation time & $200 \mathrm{~s}$ \\
Data packet size & $512 \mathrm{bytes}$ \\
Traffic application & CBR / UDP unicast \\
Transmission range & $280 \mathrm{~m}$ \\
Node speed & $0-15 \mathrm{~m} / \mathrm{s}$ \\
\hline
\end{tabular}

We collated the packet forwarding distance outputs to characterize the topologies of the simulated scenarios by node size. The protocol performance results that we had in the simulated scenarios enabled us to effectively demonstrate the proposal of the topology characterizing using packet forwarding distance measures. We primarily use the PDR outcome to describe the advantage of the multigreedy application for tasks performance in the VANET scenario of emphasis in this study.

\subsection{The packet forwarding distance averages}

Figure 2 shows the averages of the greedy packet forwarding distance measures against different node sizes in the simulated VANET environment for both the ELLIPSOID and the GREEDY methods. The average packet forwarding distance for ELLIPSOID decreases while that of GREEDY increases with increasing node size. The linear gradient patterns of the trendlines of the respective performance outcomes depict a correlation between the average packet forwarding distance and changes in node size. Figure 3 shows that the results for the RWP MANET environment are parallels to those of the VANET shown in Figure 2, which generalizes the characteristics of the ELLIPSOID and the GREEDY packet forwarding distance feature in relation to network node size.

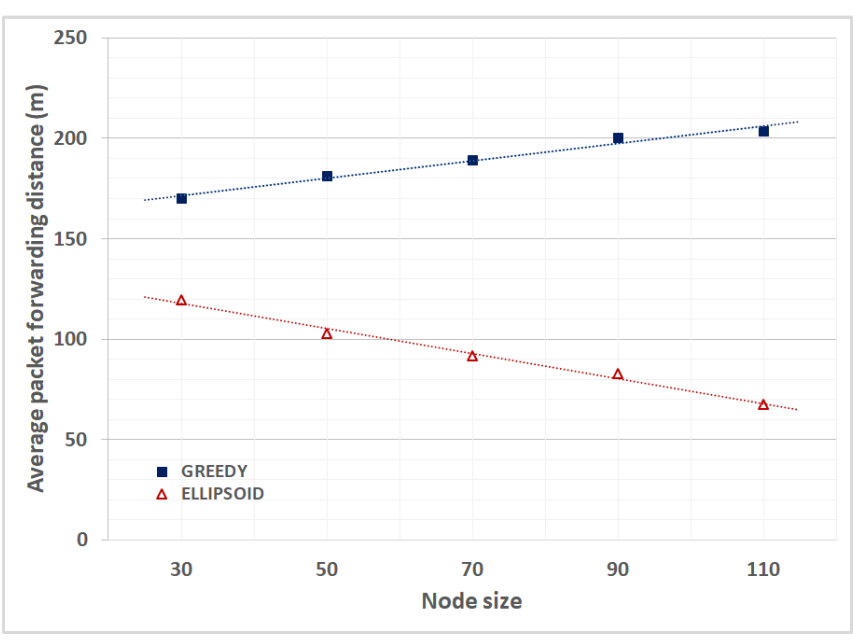

Figure 2. Packet forwarding distance averages with trendlines (VANET case).

The overall result shows the generalizability of the topology characterizing approach using the packet forwarding distance of the greedy methods and based on node size. If we look at the performance gradient of each method in isolation, such as GREEDY in Figure 2 or 3, the differences in the consecutive average packet forwarding distance across node sizes serve as potential dissimilarity indices. However, the dissimilarity measures that support multigreedy forwarding which we focus on in this paper is the pairwise differences between the ELLIPSOID and the 
GREEDY methods at each node-size level. We discuss in the next subsections the dissimilarity measurements for each of the results in Figures 2 and 3.

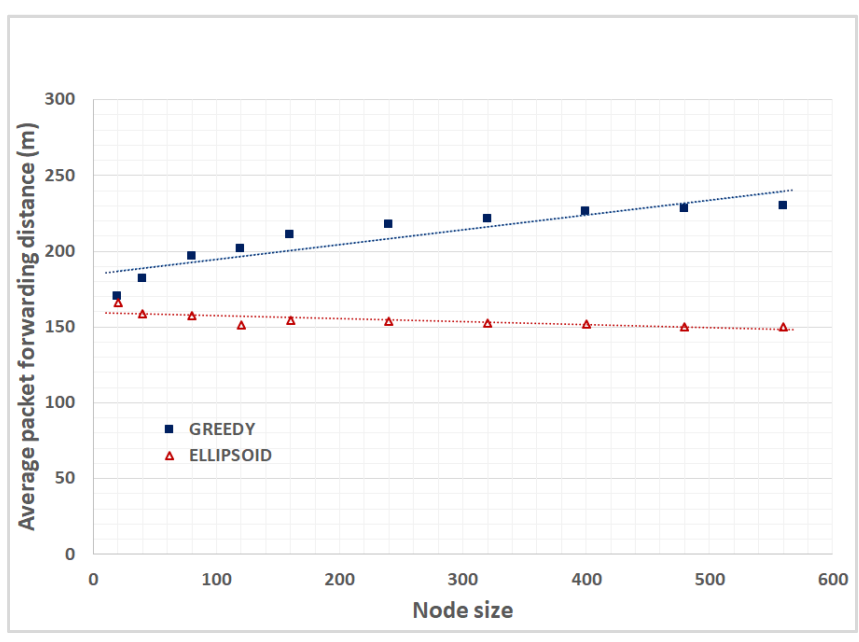

Figure 3. Packet forwarding distance averages with trendlines (MANET case).

\subsection{Dissimilarity characteristics of topology: the VANET case}

We show in Table 2 the computed dissimilarity values and the corresponding differential factors for the simulated VANET scenario.

Table 2. Topology character of the VANET environment

\begin{tabular}{|c|c|c|c|c|}
\hline \multirow[t]{2}{*}{$\begin{array}{c}\text { Node } \\
\text { size }(k)\end{array}$} & \multicolumn{2}{|c|}{$\begin{array}{l}\text { Average packet forwarding } \\
\text { distance }\end{array}$} & \multirow[t]{2}{*}{$\begin{array}{c}\text { Dissimilarity }^{4} \\
\text { value }\left(b^{(k)}\right)\end{array}$} & \multirow[t]{2}{*}{$\begin{array}{l}\text { Differential } \\
{ }^{5} \text { Factor }(d f)\end{array}$} \\
\hline & ELLIPSOID & GREEDY & & \\
\hline 30 & 119.57 & 169.87 & 50.30 & 2.70 \\
\hline 50 & 103.08 & 181.00 & 77.92 & 1.75 \\
\hline 70 & 91.69 & 189.24 & 97.55 & 1.39 \\
\hline 90 & 83.09 & 200.14 & 117.05 & 1.16 \\
\hline 110 & 67.39 & 203.38 & 135.99 & 1.00 \\
\hline
\end{tabular}

\footnotetext{
${ }^{4} b^{(k)}=\left|\bar{d}^{(E L L I P S O I D)(k)}-\bar{d}^{(G R E E D Y)(k)}\right|$
}

${ }^{5} d f\left(b^{(k)}, b^{(m)}\right)=\frac{b^{(m)}}{b^{(k)}}, b^{(m)}=135.99$.
Figure 4 shows the dissimilarity values (VANET case) from Table 2, plotted as the solid line chart, while the dashed chart lines in the lower part shows the commensurate PDR measures for the ELLIPSOID and the GREEDY methods by the different node sizes. For example, at the node size of 70 , the PDR for the ELLIPSOID method is lower at $36 \%$ compared to that of GREEDY at 54\%. But at the node size of 90 the PDR performance of ELLIPSOID is higher, 67\%, compared to the $53 \%$ of the GREEDY method. Therefore, with such additional knowledge, a routing protocol can be switching the greedy metrics based on each method's optimal PDR performance capability in relation to network node-size variations as associated with the dissimilarity values.

The differential factor values in Table 2 also give a vivid picture of the dissimilarity characteristic of the ELLIPSOID and the GREEDY methods' packet forwarding distance in the simulated VANET. The multi-greedy application would be advantageous in this VANET environment, as shown by the distinctiveness in the relative ratios of the differential factors having significant margins of difference one from the other.

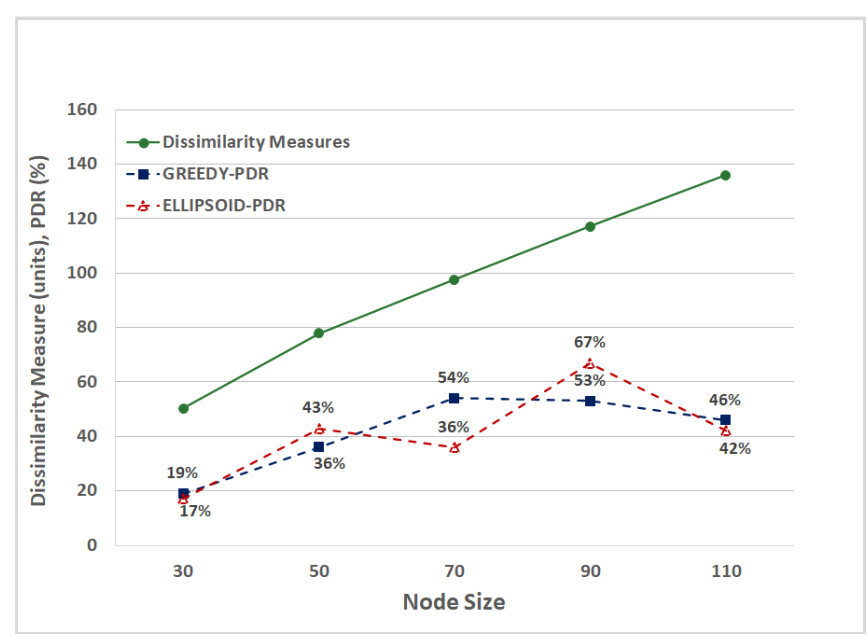

Figure 4. Dissimilarity distribution of the packet forwarding distance averages; and the PDR. (VANET case)

Figures 5 and 6 respectively show the routing protocol's average hop count and the average delay performances of the VANET scenario. The unevenness of the hop count and the delay performance indications could be leveraged for improved forwarding performance, using the multi-greedy approach. As expected, GREEDY performed with a lower hop count in comparison to ELLIPSOID for all node sizes. Nevertheless, at node sizes 50 and 90 where ELLIPSOID has higher PDRs (Figure 4), a multi-greedy method may implement a trade-off that determines which of the two greedy methods would perform optimally for the next relay selection. The average delay for both methods at node-size 
90 is at about the same level of $15 \mathrm{~ms}$, which supports the method of choice to be ELLIPSOID with its 67\% PDR against $53 \%$ of GREEDY's. Thus, in VANET scenarios of this sort, the dissimilarity characterizing of topology, based on the average packet forwarding distance comparisons offers potential support for efficient multi-greedy routing involving the ELLIPSOID and the GREEDY methods.

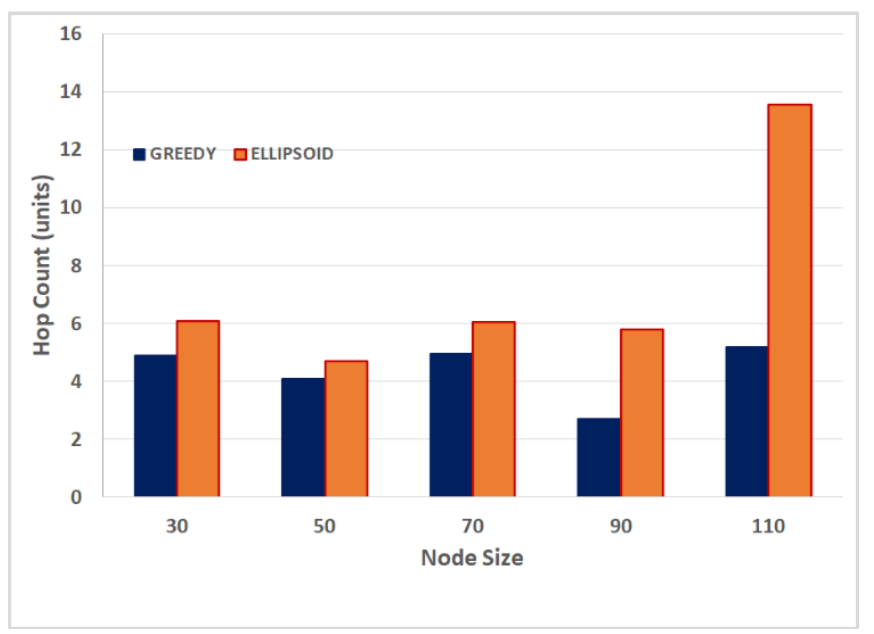

Figure 5. Average hop count (VANET case)

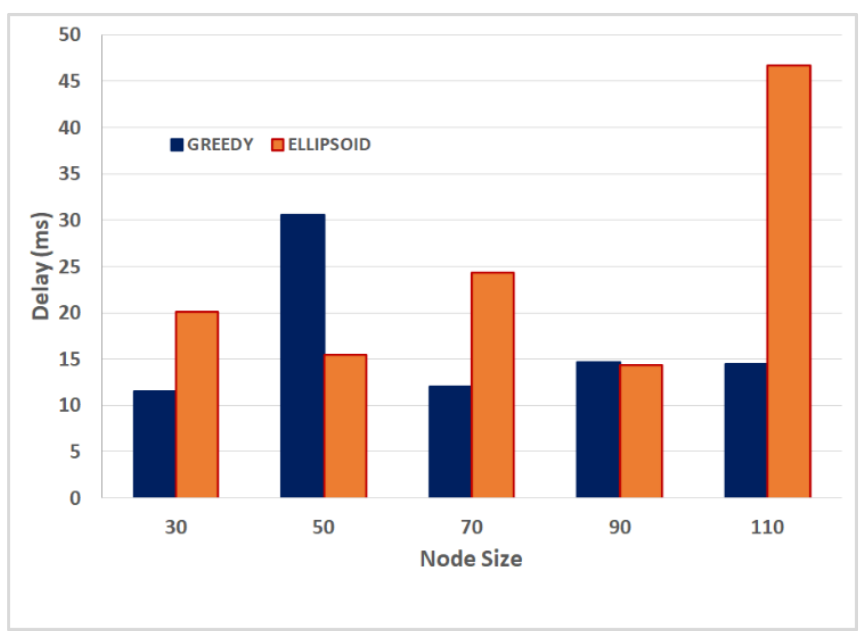

Figure 6. Average delay (VANET case)

\subsection{Dissimilarity characteristics of topology: the MANET case}

We show in Table 3 the computed dissimilarity values and the corresponding differential factors for the simulated MANET scenario. Figure 7 shows the dissimilarity values (MANET case) from Table 3 plotted as the solid line chart, while the dashed line charts above it are the corresponding protocol PDR performances for both the ELLIPSOID and the GREEDY at the respective node sizes. Generally, the protocol performance of both methods is quite high in this network.

Table 3. Topology character of the MANET environment

\begin{tabular}{ccccc}
\hline \hline $\begin{array}{c}\text { Node } \\
\text { Size }(k)\end{array}$ & \multicolumn{2}{c}{$\begin{array}{c}\text { Average packet forwarding } \\
\text { distance }\end{array}$} & $\begin{array}{c}\text { Dissimilarity } \\
\text { Value }(b)\end{array}$ & $\begin{array}{c}\text { Differential } \\
\text { Factor }(d f)\end{array}$ \\
\hline 20 & 166.09 & 170.27 & 4.18 & 19.11 \\
40 & 158.76 & 181.94 & 23.18 & 3.45 \\
80 & 157.50 & 196.62 & 39.12 & 2.04 \\
120 & 151.25 & 201.75 & 50.50 & 1.58 \\
160 & 154.61 & 210.75 & 56.14 & 1.42 \\
240 & 153.62 & 217.50 & 63.88 & 1.25 \\
320 & 152.31 & 221.49 & 69.18 & 1.15 \\
400 & 152.16 & 226.12 & 73.96 & 1.08 \\
480 & 150.11 & 228.20 & 78.09 & 1.02 \\
560 & 150.28 & 230.18 & 79.90 & 1.00 \\
\hline
\end{tabular}

In comparison to the VANET's case (Figure 4) that appears linear, the gradient of the dissimilarity values of the MANET case (Figure 4) is more curved and appears to reach an asymptotic level at the point of very high node sizes. At high levels of node size, the rate of change of the dissimilarity curve evens out.

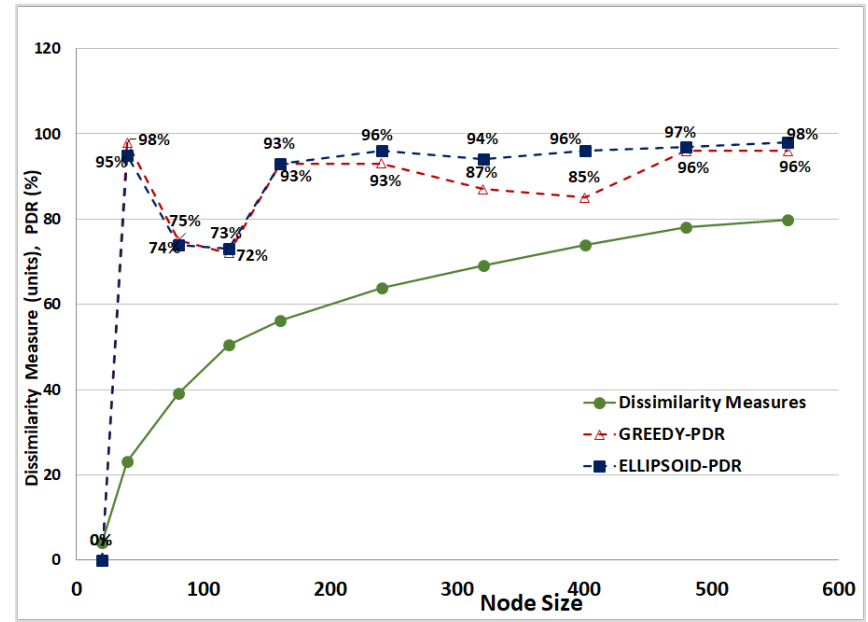

Figure 7. Dissimilarity distribution of the packet forwarding distance averages; and the PDR. (MANET case) 
Equally, the PDR performances can be seen to be levelling out at high node sizes. The PDR performance across the different node sizes, at the 40 mark and from 180 to 560 , is above $90 \%$. These outcomes suggest that the multi-greedy method may not have an advantage in the MANET environment depicted; except regarding the hop count and the delay values as shown in Figures 8 and 9. There were no packets delivered for the node size of 20, although there was some forwarding performance for both methods.

The differential factor values of $1.15,1.08,1.02$, and 1.00 corresponding to $320,400,480$, and 560 node sizes respectively (Table 3) show that in this network the packet forwarding distance dissimilarity has little comparative changes at high node size levels. Specifically, the multigreedy application may not be so useful in the range beyond 500 node-size where the differential ratios are only slight margins apart. For example, the 1.02 and 1.00 for the node size 480 and 560 have a low difference of 0.02 . Generally, differential ratios with marginal differences classify the network area as having very little opportunity for multigreedy performance improvement.

Figures 8 and 9 show the hop count and the delay in routing protocol performance for the ELLIPSOID and the GREEDY forwarding at the different node sizes for the simulated MANET environment. The figures show unevenness in the average hop count and delay outcomes for both GREEDY and ELLIPSOID; hence, a multi-greedy forwarding application could improve packet delivery efficiency based on these indicators. As expected, the hopcount of GREEDY is lower in comparison to that of GREEDY at all node sizes. However, the delay of GREEDY is higher at node sizes 160 and 560 .

Generally, when viable and for a prevailing node size or dissimilarity index, the hop count and delay indications could be leveraged for multi-greedy packet forwarding improvement. When a learning method through exploration sampling [1] has determined the topology dissimilarity indices and the commensurate protocol performance indications (i.e. PDR, hop count, etc.) of a MANET, it can leverage these for improvement in the future task performances.

\section{Conclusions}

We have shown the greedy packet forwarding distance as a potential dissimilarity feature for the characterizing of MANET topologies in relation to node size, which also supports multi-greedy efficient geographic routing. By sampling the aggregates of the packet forwarding distance from the engaged greedy methods, the routing protocol can discern the distributions of the dissimilarity indices as associated with the prevailing node size of a network and adapt its performance for efficient packet forwarding. We described models for dissimilarity characterizing of topology based on the averages of the greedy packet forwarding distance measurements.

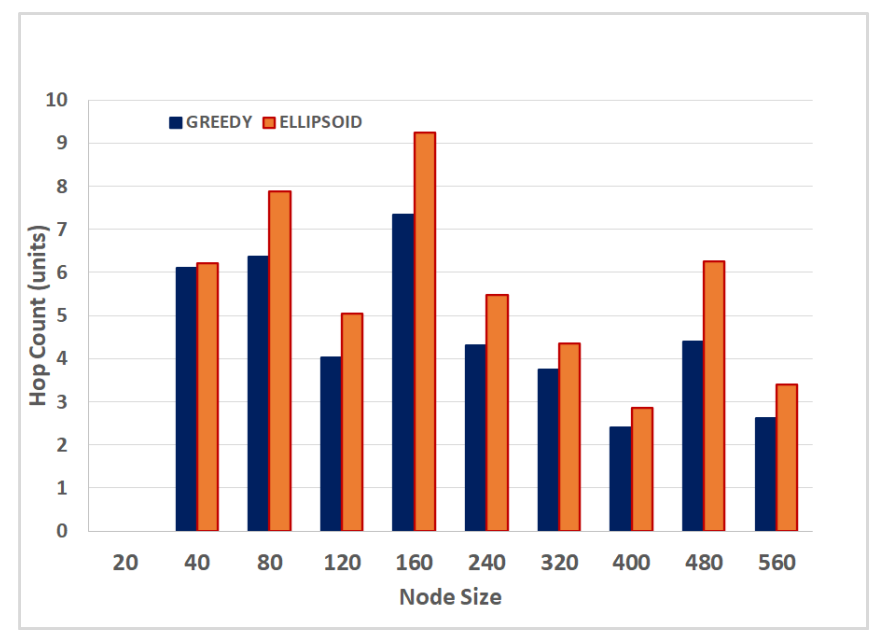

Figure 8. Average hop count (MANET case)

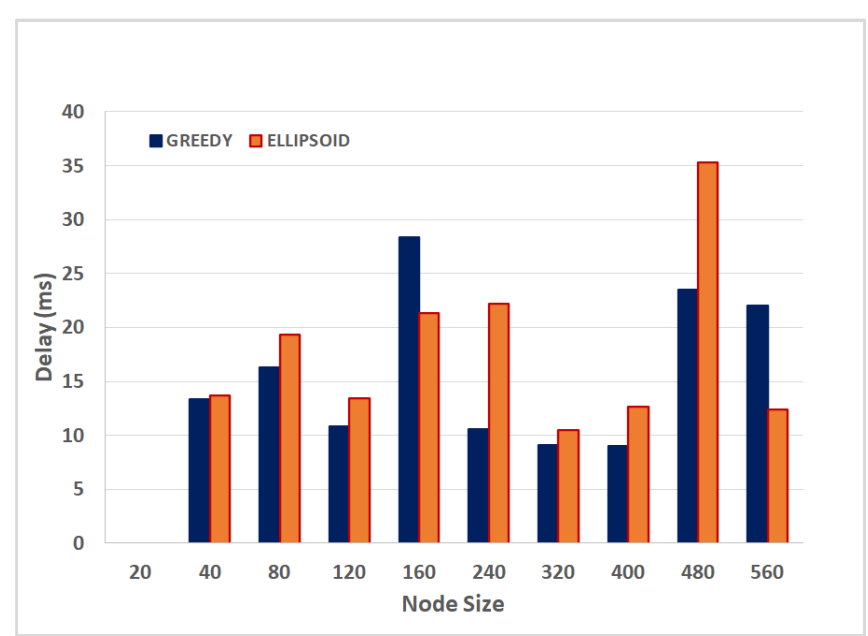

Figure 9. Average delay (MANET case)

Our experimentation with the ELLIPSOID and the GREEDY metrics demonstrated the potential for improvement in successful packets delivery using the packet forwarding distance dissimilarity for topology characterizing and adaptive multi-greedy forwarding performance. The result generally shows that as the network node size increases the GREEDY metric reflect more longer-lengthed packet forwarding distance in its distributions, while that of the ELLIPSOID is the opposite. Following the approach in [1], a learning method in geographic routing can classify distributions of the packet forwarding distance in relation to node sizes for characterizing a network. This approach to topology characterizing is quite robust and flexible, i.e. by node size variations and packet forwarding distance over a wide range of networking environments and irrespective of the underlying graph type, in contrast to the approach in [1].

The topology characterizing method that we described in this paper is most suitable for improving geographic greedy 
routing in VANETs and FANETs where node sizes exhibit periodic variations. In this work, we designated the packet forwarding distance as the weight, in length measure, of the link between a node and its next relay neighbour to which it transmits packets. Instead of a single attribute, i.e. the length measurement, in future work other link attributes, including PIR [5], transmission power cost, etc. could be factored into the dissimilarity valuations of the greedy packet forwarding distance.

\section{Acknowledgements.}

This work was supported in part by the Research Initiation Grant of the Botswana International University of Science and Technology, Project Code No. S00122.

\section{References}

[1] P. Cucka, N. S. Netanyahu, and A. Rosenfeld, "Learning in navigation: goal finding in graphs," in Studies in Pattern Recognition, vol. Volume 25, 0 vols., WORLD SCIENTIFIC, 1997, pp. 39-56. doi: 10.1142/9789812797728_0003.

[2] L. da F. Costa, F. A. Rodrigues, G. Travieso, and P. R. V. Boas, "Characterization of complex networks: A survey of measurements," Advances in Physics, vol. 56, no. 1, pp. 167-242, Jan. 2007, doi: 10.1080/00018730601170527.

[3] V. Srivastava, A. B. Hilal, M. S. Thompson, J. N. Chattha, A. B. MacKenzie, and L. A. DaSilva, "Characterizing mobile ad hoc networks -: the maniac challenge experiment," in Proceedings of the third ACM international workshop on Wireless network testbeds, experimental evaluation and characterization, San Francisco, California, USA, Sep. 2008, pp. 65-72. doi: 10.1145/1410077.1410090.

[4] D. Medina, F. Hoffmann, S. Ayaz, and C.-H. Rokitansky, "Topology characterization of high density airspace aeronautical ad hoc networks," in 2008 5th IEEE International Conference on Mobile Ad Hoc and Sensor Systems, Sep. 2008, pp. 295-304. doi: 10.1109/MAHSS.2008.4660016.

[5] F. Lyu et al., "Characterizing Urban Vehicle-to-Vehicle Communications for Reliable Safety Applications," IEEE Transactions on Intelligent Transportation Systems, vol. 21, no. 6 , pp. 2586-2602, Jun. 2020, doi: 10.1109/TITS.2019.2920813.

[6] A. Silva, N. Reza, and A. Oliveira, "Improvement and Performance Evaluation of GPSR-Based Routing Techniques for Vehicular Ad Hoc Networks," IEEE Access, vol. 7, pp. 21722-21733, 2019, doi: 10.1109/ACCESS.2019.2898776.

[7] T. Ducrocq, M. Hauspie, N. Mitton, and S. Pizzi, "On the Impact of Network Topology on Wireless Sensor Networks Performances: Illustration with Geographic Routing," in 2014 28th International Conference on Advanced Information Networking and Applications Workshops, May 2014, pp. 719-724. doi: 10.1109/WAINA.2014.118.

[8] R. P. W. Duin and E. Pękalska, "The dissimilarity space: Bridging structural and statistical pattern recognition," Pattern Recognition Letters, vol. 33, no. 7, pp. 826-832, May 2012, doi: 10.1016/j.patrec.2011.04.019.

[9] L. da F. Costa et al., "Analyzing and modeling real-world phenomena with complex networks: a survey of applications," Advances in Physics, vol. 60, no. 3, pp. 329412, Jun. 2011, doi: 10.1080/00018732.2011.572452.

[10] Y. M. G. Costa, D. Bertolini, A. S. Britto, G. D. C. Cavalcanti, and L. E. S. Oliveira, "The dissimilarity approach: a review," Artif Intell Rev, vol. 53, no. 4, pp. 2783-2808, Apr. 2020, doi: 10.1007/s10462-019-09746-z.

[11] R. C. Veltkamp, "Shape matching: similarity measures and algorithms," in Proceedings International Conference on Shape Modeling and Applications, May 2001, pp. 188-197. doi: 10.1109/SMA.2001.923389.

[12] J. Kuruvila, A. Nayak, and I. Stojmenovic, "Hop count optimal position based packet routing algorithms for ad hoc wireless networks with a realistic physical layer," in 2004 IEEE International Conference on Mobile Ad-hoc and Sensor Systems (IEEE Cat. No.04EX975), Oct. 2004, pp. 398-405. doi: 10.1109/MAHSS.2004.1392179.

[13] G. Oladeji-Atanda, D. Mpoeleng, and F. J. Ogwu, "Multigreedy geographic packets forwarding using flow-based indicators," EAI-MCA, vol. 6, no. 18, May 2021, doi: 10.4108/eai.13-5-2021.169914.

[14] P. Bose et al., "Online routing in convex subdivisions," Int. J. Comput. Geom. Appl., vol. 12, no. 04, pp. 283-295, Aug. 2002, doi: 10.1142/S021819590200089X.

[15] S. Faye and C. Chaudet, "Characterizing the Topology of an Urban Wireless Sensor Network for Road Traffic Management," IEEE Transactions on Vehicular Technology, vol. 65, no. 7, pp. 5720-5725, Jul. 2016, doi: 10.1109/TVT.2015.2465811

[16] G. S.-C. Kao, T. Fevens, and J. Opatrny, "Position-based routing on 3-D geometric graphs in mobile ad hoc networks," in 17th Canadian Conference on Computational Geometry (CCCG'05), Windsor, Ontario, 2005, pp. 81-91. [Online]. Available: http://www.cccg.ca/proceedings/2005/

[17] K. Yamazaki and K. Sezaki, "A proposal of geographical routing protocols for location-aware services," Electronics and Communications in Japan (Part I: Communications), vol. 87, no. 4, pp. 26-34, Apr. 2004, doi: 10.1002/ecja.10138.

[18] A. Bothe and N. Aschenbruck, "BonnMotion 4 - Taking Mobility Generation to the Next Level," in 2020 IEEE 39th International Performance Computing and Communications Conference (IPCCC), Nov. 2020, pp. 1-8. doi: 10.1109/IPCCC50635.2020.9391563.

[19] A. Bujari, C. E. Palazzi, and D. Ronzani, "A Comparison of Stateless Position-based Packet Routing Algorithms for FANETs," IEEE Transactions on Mobile Computing, vol. 17, no. 11, pp. 2468-2482, Nov. 2018, doi: 10.1109/TMC.2018.2811490. 